\title{
A Hardy space related to the square root of the Poisson kernel
}

by

JonATAN VASILIS (Göteborg)

\begin{abstract}
A real-valued Hardy space $H_{\sqrt{v}}^{1}(\mathbb{T}) \subseteq L^{1}(\mathbb{T})$ related to the square root of the Poisson kernel in the unit disc is defined. The space is shown to be strictly larger than its classical counterpart $H^{1}(\mathbb{T})$. A decreasing function is in $H_{\sqrt{ }}^{1}(\mathbb{T})$ if and only if the function is in the Orlicz space $L \log \log L(\mathbb{T})$. In contrast to the case of $H^{1}(\mathbb{T})$, there is no such characterization for general positive functions: every Orlicz space strictly larger than $L \log L(\mathbb{T})$ contains positive functions which do not belong to $H_{\sqrt{ }}^{1}(\mathbb{T})$, and no Orlicz space of type $\Delta_{2}$ which is strictly smaller than $L^{1}(\mathbb{T})$ contains every positive function in $H_{\sqrt{ }}^{1}(\mathbb{T})$. Finally, we have a characterization of certain eigenfunctions of the hyperbolic Laplace operator in terms of $H_{\sqrt{V}}^{1}(\mathbb{T})$.
\end{abstract}

1. Introduction. Let $\mathbb{T}$ denote the unit circle in $\mathbb{R}^{2}$, which we identify with angles in $\mathbb{R} / 2 \pi \mathbb{Z}$. The classical real-valued Hardy space $H^{1}(\mathbb{T})$ can be defined as the set of $f \in L^{1}(\mathbb{T})$ such that the radial maximal function $P^{*} f$ is in $L^{1}(\mathbb{T})$. Here $\left(P^{*} f\right)(\theta)=\sup _{r \in[0,1[}|(P f)(r, \theta)|, \theta \in \mathbb{T}$, and $P f$ is the Poisson extension of $f$, that is, $(P f)(r, \theta)=\int_{\mathbb{T}} P(r, \theta-\phi) f(\phi) d \phi$ for $r \in$ $[0,1[$ and $\theta \in \mathbb{T}$, where

$$
P(r, \theta)=\frac{1}{2 \pi} \cdot \frac{1-r^{2}}{1-2 r \cos \theta+r^{2}}
$$

is the Poisson kernel in the unit disc. We are instead interested in the space corresponding to the square root of the Poisson kernel, defined as follows. For $f \in L^{1}(\mathbb{T}), r \in\left[0,1\left[\right.\right.$ and $\theta \in \mathbb{T}$ we let $P_{0}(r, \theta)=\sqrt{P(r, \theta)}$ and define

$$
\begin{aligned}
\left(P_{0} f\right)(r, \theta) & =\int_{\mathbb{T}} P_{0}(r, \theta-\phi) f(\phi) d \phi, \\
\left(\mathcal{P}_{0} f\right)(r, \theta) & =\frac{\left(P_{0} f\right)(r, \theta)}{\left(P_{0} 1\right)(r, 0)}, \\
\left(\mathcal{P}_{0}^{*} f\right)(\theta) & =\sup _{r \in[0,1[}\left|\left(\mathcal{P}_{0} f\right)(r, \theta)\right|,
\end{aligned}
$$

2010 Mathematics Subject Classification: Primary 42B30; Secondary 42B25, 31A10. Key words and phrases: Hardy space, Poisson kernel, $L \log \log L$. 
where, as opposed to the classical case, we must normalize by dividing by the radial function $P_{0} 1$. A Hardy type space related to $\mathcal{P}_{0}^{*}$ is now defined just as in the classical case.

Definition. The real-valued Hardy space $H_{\sqrt{v}}^{1}(\mathbb{T})$ is the subspace of $L^{1}(\mathbb{T})$ consisting of functions $f \in L^{1}(\mathbb{T})$ such that $\mathcal{P}_{0}^{*} f \in L^{1}(\mathbb{T})$. A norm $\|\cdot\|_{H_{V}^{1}(\mathbb{T})}$ on $H_{\sqrt{V}}^{1}(\mathbb{T})$ is defined by $\|f\|_{H_{\sqrt{ }}^{1}(\mathbb{T})}=\left\|\mathcal{P}_{0}^{*} f\right\|_{L^{1}(\mathbb{T})}$.

Our interest in studying how classical results change when the ordinary Poisson operator $P$ is replaced by $\mathcal{P}_{0}$ stems from the article [23] by Sjögren. Classically, Fatou's theorem on boundary convergence states that if $f \in$ $L^{1}(\mathbb{T})$, then $(P f)(r, \theta) \rightarrow f(\alpha)$ almost everywhere as $(r, \theta) \rightarrow\left(1^{-}, \alpha\right)$ nontangentially, in the sense that $|\theta-\alpha| /(1-r)$ remains bounded. Replacing $P$ by $\mathcal{P}_{0}$, we get a similar result $\left[23\right.$ : if $f \in L^{1}(\mathbb{T})$, then $\left(\mathcal{P}_{0} f\right)(r, \theta) \rightarrow f(\alpha)$ almost everywhere as $(r, \theta) \rightarrow\left(1^{-}, \alpha\right)$ weakly tangentially, meaning that $|\theta-\alpha| /\left((1-r) \log \left(\frac{2}{1-r}\right)\right)$ remains bounded. That is, the operator $\mathcal{P}_{0}$ allows us to extend the approach region given by Fatou's theorem - which is sharp for $P$ by a theorem due to Littlewood [13]. For $\mathcal{P}_{0}$ there are several results on sharp approach regions for boundary functions $f$ in other function spaces, including $L^{p}(\mathbb{T})$ for $1 \leq p \leq+\infty$ and weak $L^{p}(\mathbb{T})$ for $1<p<+\infty$; see [2, 3, 19, 24]. There are also similar results in other settings [4, 5, 16, 20, 21, 22, 26].

Interest in the operator $\mathcal{P}_{0}$ itself comes from the fact that every eigenfunction of the hyperbolic Laplace operator, $\frac{1}{4}\left(1-r^{2}\right)^{2} \Delta$, admits an integral representation in terms of a power of the Poisson kernel [10, 12]. The exponent is determined by the eigenvalue, and the square root is the case giving the smallest possible eigenvalue with positive eigenfunctions.

Finally, note that $f \in H^{1}(\mathbb{T})$ obviously does not imply that $|f| \in H^{1}(\mathbb{T})$, and that the same is true for $H_{\sqrt{V}}^{1}(\mathbb{T})$. That is, whether a function is in the Hardy space or not may depend on cancellation between the positive and the negative parts. Nevertheless, studying the positive functions - as we will do - is of interest also in the classical case; see for instance [14].

2. Results. The space $H_{\sqrt{ }}^{1}(\mathbb{T})$ is strictly larger than its classical counterpart $H^{1}(\mathbb{T})$, with the inclusion given by the following proposition.

Proposition 2.1. $H^{1}(\mathbb{T}) \subseteq H_{\sqrt{ }}^{1}(\mathbb{T})$, and there exists a constant $C>0$ such that $\|f\|_{H_{\checkmark}^{1}(\mathbb{T})} \leq C\|f\|_{H^{1}(\mathbb{T})}$ for all $f \in H^{1}(\mathbb{T})$.

For positive functions $f \in L^{1}(\mathbb{T}), f \geq 0$, it is well known in the classical case - see for instance [25] or [8, Section II.2] - that $f \in H^{1}(\mathbb{T})$ if and only if $f \in L \log L(\mathbb{T})$. It is easy to see that this equivalence also holds if, instead of positivity, we require that $f \in L^{1}(\mathbb{T})$ be decreasing, as defined in 
Proposition 2.2. Our next result states that in the case of $H_{\sqrt{ }}^{1}(\mathbb{T})$, a similar equivalence - with an iterated logarithm - holds for decreasing functions.

Proposition 2.2. Assume that $f \in L^{1}(\mathbb{T})$. If both $f(\zeta+\theta)$ and $f(\zeta-\theta)$ are decreasing functions of $\theta \in\left[0, \pi\left[\right.\right.$ for some fixed $\zeta \in \mathbb{T}$, then $f \in H_{\sqrt{ }}^{1}(\mathbb{T})$ if and only if $f \in L \log \log L(\mathbb{T})$.

The Orlicz spaces $L \log L(\mathbb{T})$ and $L \log \log L(\mathbb{T})$ are defined in the next section.

The equivalence of Proposition 2.2 does not, however, extend to general positive functions. In fact, our main result (Theorem 2.3) states that neither implication can hold: although $H_{\sqrt{V}}^{1}(\mathbb{T})$ contains $L \log L(\mathbb{T})$, it contains no strictly larger Orlicz space, and conversely, $L^{1}(\mathbb{T})$ is the smallest Orlicz space of type $\Delta_{2}$ that contains all, or all positive, functions in $H_{\sqrt{ }}^{1}(\mathbb{T})$.

TheOREM 2.3. Let $\Phi:[0,+\infty[\rightarrow[0,+\infty[$ be a convex and increasing function satisfying $\Phi(0)=0$ and $\Phi(x) \rightarrow+\infty$ as $x \rightarrow+\infty$. Denote by $L^{\Phi}(\mathbb{T})$ the corresponding Orlicz space.

(i) If $\liminf _{x \rightarrow+\infty} \Phi(x) /(x \log x)=0$, then there exists a function $f \in$ $L^{\Phi}(\mathbb{T}), f \geq 0$, such that $f \notin H_{\sqrt{V}}^{1}(\mathbb{T})$.

(ii) If $\Phi$ is of type $\Delta_{2}$ - that is, if $\Phi(2 x) \leq C \Phi(x)$ for all sufficiently large $x$-and $\lim \sup _{x \rightarrow+\infty} \Phi(x) / x=+\infty$, then there exists a function $g \in H_{\sqrt{V}}^{1}(\mathbb{T}), g \geq 0$, such that $g \notin L^{\Phi}(\mathbb{T})$.

Just as in the classical case, the space $H_{\sqrt{ }}^{1}(\mathbb{T})$ does not change if we a priori allow distributions rather than just $L^{1}(\mathbb{T})$-functions in the definition. That is, defining $\mathcal{P}_{0}^{*} T$ for distributions $T \in \mathcal{D}^{\prime}(\mathbb{T})$ in the obvious way (see Section 4.3), we prove that $\mathcal{P}_{0}^{*} T \in L^{1}(\mathbb{T})$ implies that $T$ is actually a function in $L^{1}(\mathbb{T})$.

Proposition 2.4. Let $T \in \mathcal{D}^{\prime}(\mathbb{T})$. If $\mathcal{P}_{0}^{*} T \in L^{1}(\mathbb{T})$ then $T \in L^{1}(\mathbb{T})$.

Finally, we have a characterization of certain eigenfunctions of the hyperbolic Laplace operator in terms of $H_{\sqrt{ }}^{1}(\mathbb{T})$.

Proposition 2.5. Let $\mathbb{D}$ be the unit disc in $\mathbb{R}^{2}$. If $u \in C^{2}(\mathbb{D})$ satisfies

(i) $\frac{1}{4}\left(1-r^{2}\right)^{2} \Delta u(r, \theta)=-\frac{1}{4} u(r, \theta)$ in $\mathbb{D}$, and

(ii) $u^{*} \in L^{1}(\mathbb{T})$, where $u^{*}(\theta)=\sup _{r \in[0,1[} \frac{|u(r, \theta)|}{\left(P_{0} 1\right)(r, 0)}$,

then there exists a function $f \in H_{\sqrt{V}}^{1}(\mathbb{T})$ such that $u=P_{0} f$. Conversely, if $f \in H_{\sqrt{ }}^{1}(\mathbb{T})$ then $u=P_{0} f$ defines a function satisfying (i) and (ii). 
3. Preliminaries. As usual we identify the unit circle $\mathbb{T}$ with angles in $\mathbb{R} / 2 \pi \mathbb{Z}$, and we let $|\theta|$ denote the modulus of the representative for $\theta \in \mathbb{T}$ in the interval $]-\pi, \pi]$. Unless otherwise specified, integration over subsets of $\mathbb{T}$ is always with respect to the rotationally invariant arc measure of $\mathbb{T}$, normalized so that $\mathbb{T}$ has measure $2 \pi$. Suitably interpreted, this coincides with ordinary Lebesgue measure on the real line, and we let $|E|$ denote the Lebesgue measure of a measurable set $E \subseteq \mathbb{R}$. Identifying a measurable set $E \subseteq \mathbb{T}$ with a subset in $\mathbb{R}$, we have $|E|=\int_{E} d \theta$, where the integration is in $\mathbb{T}$.

The symbol $C$ will denote a sufficiently large, strictly positive but finite constant whose exact value can change even within the same line. Similarly $c$ denotes a small, strictly positive constant; that is, $c=C^{-1}$. Given functions $f$ and $g$ satisfying $c g \leq f \leq C g$ pointwise, we write $f \sim g$. For $x \in \mathbb{R}$, we let $\lfloor x\rfloor$ denote the largest integer less than or equal to $x$.

In the introduction we defined the classical Hardy space $H^{1}(\mathbb{T})$ to be the set of $f \in L^{1}(\mathbb{T})$ such that $P^{*} f \in L^{1}(\mathbb{T})$. Equivalently, this space can be defined in terms of atoms [6], namely

$$
H^{1}(\mathbb{T})=\left\{\sum_{j \geq 0} \lambda_{j} a_{j} ; \sum_{j \geq 0}\left|\lambda_{j}\right|<+\infty \text { and } a_{0}, a_{1}, \ldots \text { are atoms }\right\},
$$

where $\sum_{j \geq 0} \lambda_{j} a_{j}$ converges in $L^{1}(\mathbb{T})$. An atom is a function $a \in L^{\infty}(\mathbb{T})$ which is either the constant $\frac{1}{2 \pi}$, or is such that for some interval $I \subseteq \mathbb{T}$,

(i) $\operatorname{supp} a \subseteq I$,

(ii) $\|a\|_{L^{\infty}(\mathbb{T})} \leq 1 /|I|$,

(iii) $\int_{\mathbb{T}} a(\theta) d \theta=0$.

A norm on $H^{1}(\mathbb{T})$ is defined by $\|f\|_{H^{1}(\mathbb{T})}=\inf \sum_{j \geq 0}\left|\lambda_{j}\right|$, where the infimum is over all atomic decompositions $\sum_{j \geq 0} \lambda_{j} a_{j}$ of $f$.

By an Orlicz space on $\mathbb{T}$, denoted $L^{\Phi}(\mathbb{T})$, we mean the set of all measurable functions $f: \mathbb{T} \rightarrow \mathbb{R}$ such that $\int_{\mathbb{T}} \Phi(\alpha|f(\theta)|) d \theta<+\infty$ for some constant $\alpha>0$, where $\Phi:[0,+\infty[\rightarrow[0,+\infty[$ is a given convex and increasing function such that $\Phi(0)=0$ and $\Phi(x) \rightarrow+\infty$ as $x \rightarrow+\infty$. As usual, two functions in $L^{\Phi}(\mathbb{T})$ are identified if they are equal almost everywhere. Since $\Phi$ is increasing and convex it follows that the space $L^{\Phi}(\mathbb{T})$ is a vector space. We say that $L^{\Phi}(\mathbb{T})$ and $\Phi$ are of type $\Delta_{2}$ if $\Phi(2 x) \leq C \Phi(x)$ for all sufficiently large $x$. In this case a measurable function $f$ is in $L^{\Phi}(\mathbb{T})$ if and only if $\int_{\mathbb{T}} \Phi(f(\theta)) d \theta<+\infty$. We refer to [18] for a background on Orlicz spaces.

Two Orlicz spaces will be of particular interest to us: the classical space $L \log L(\mathbb{T})$, which is the Orlicz space $L^{\Phi}(\mathbb{T})$ with $\Phi(x)=x \log (1+x), x \geq 0$, and the space $L \log \log L(\mathbb{T})$, defined as follows. 
Definition. The space $L \log \log L(\mathbb{T})$ is the Orlicz space $L^{\Phi}(\mathbb{T})$ with $\Phi(x)=x \log (1+\log (1+x)), x \geq 0$.

Clearly, both spaces are of type $\Delta_{2}$ and are subspaces of $L^{1}(\mathbb{T})$.

\section{Proofs}

4.1. The inclusion $H^{1}(\mathbb{T}) \subseteq H_{\sqrt{ }}^{1}(\mathbb{T})$. The main step of the proof of Proposition 2.1 is to show that the atoms have uniformly bounded norm in $H_{\sqrt{ }}^{1}(\mathbb{T})$.

Lemma 4.1. There exists a constant $C>0$ such that $\|a\|_{H_{\sqrt{V}}^{1}(\mathbb{T})} \leq C$ for all atoms $a$.

The normalizing factor $\left(P_{0} 1\right)(r, 0)$ satisfies

$$
\left(P_{0} 1\right)(r, 0) \sim(1-r)^{1 / 2} \log \left(\frac{2}{1-r}\right)
$$

(see [23]), valid for all $r \in[0,1[$.

Proof of Lemma 4.1. If $a$ is the constant atom, then obviously $\left\|\mathcal{P}_{0}^{*} a\right\|_{L^{1}(\mathbb{T})}$ $\leq C$. Otherwise $\int_{\mathbb{T}} a(\theta) d \theta=0$ and there exist $\zeta \in \mathbb{T}$ and $\left.\left.s \in\right] 0, \pi\right]$ such that $\operatorname{supp} a \subseteq\{\eta \in \mathbb{T} ;|\zeta-\eta| \leq s\}$ and $\|a\|_{L^{\infty}(\mathbb{T})} \leq 1 /(2 s)$. Without loss of generality we may assume that $\zeta=0$.

To show that $\left\|\mathcal{P}_{0}^{*} a\right\|_{L^{1}(\mathbb{T})} \leq C$, we first consider integration over $\theta \in \mathbb{T}$ with $|\theta| \leq 2 s$. We have $\left|\left(\mathcal{P}_{0} a\right)(r, \theta)\right| \leq\|a\|_{L^{\infty}(\mathbb{T})} \leq 1 /(2 s)$, and hence

$$
\int_{|\theta| \leq 2 s} \sup _{r \in[0,1[}\left|\left(\mathcal{P}_{0} a\right)(r, \theta)\right| d \theta \leq C .
$$

For the case when $|\theta|>2 s$, we make use of the moment condition $\int_{\mathbb{T}} a(\phi) d \phi=0$ by subtracting $P_{0}(r, \theta)$ when estimating $\left(\mathcal{P}_{0} a\right)(r, \theta)$ :

$$
\begin{aligned}
\left|\left(\mathcal{P}_{0} a\right)(r, \theta)\right| & =\frac{1}{\left(P_{0} 1\right)(r, 0)}\left|\int_{\mathbb{T}}\left(P_{0}(r, \theta-\phi)-P_{0}(r, \theta)\right) a(\phi) d \phi\right| \\
& \leq \frac{C}{(1-r)^{1 / 2} \log \left(\frac{2}{1-r}\right)} \int_{\mathbb{T}}\left|P_{0}(r, \theta-\phi)-P_{0}(r, \theta)\right| \cdot|a(\phi)| d \phi .
\end{aligned}
$$

Letting $D_{2} P_{0}$ denote the partial derivative of $P_{0}$ with respect to the second variable, the mean value theorem gives

$$
\left|P_{0}(r, \theta-\phi)-P_{0}(r, \theta)\right|=\left|\left(D_{2} P_{0}\right)(r, \xi)\right| \cdot|\phi|,
$$

where $|\phi| \leq s$ and $\xi$ is some point on the shortest path between $\theta-\phi$ and $\theta$, and since $|\phi| \leq s<|\theta| / 2$ we have $|\xi| \sim|\theta|$. Since

$$
\left|\left(D_{2} P_{0}\right)(r, \xi)\right|=\frac{1}{\sqrt{2 \pi}} \cdot r\left(1-r^{2}\right)^{1 / 2} \frac{|\sin \xi|}{\left(1-2 r \cos \xi+r^{2}\right)^{3 / 2}}
$$


we get

$$
\left|\left(\mathcal{P}_{0} a\right)(r, \theta)\right| \leq \int_{\mathbb{T}} \frac{C s|\sin \xi|}{\log \left(\frac{2}{1-r}\right)\left(1-2 r \cos \xi+r^{2}\right)^{3 / 2}} \cdot|a(\phi)| d \phi,
$$

and if $|\xi|<\pi / 2$, then $|\sin \xi| \sim|\xi| \sim|\theta|$ and $1-\cos \xi \sim|\xi|^{2} \sim|\theta|^{2}$, so that

$$
\left|\left(\mathcal{P}_{0} a\right)(r, \theta)\right| \leq \frac{C s|\theta|}{\log \left(\frac{2}{1-r}\right)\left((1-r)^{2}+c r|\theta|^{2}\right)^{3 / 2}} \int_{\mathbb{T}}|a(\phi)| d \phi \leq \frac{C s}{|\theta|^{2}} .
$$

If instead $|\xi| \geq \pi / 2$, then $\left|\left(D_{2} P_{0}\right)(r, \xi)\right| /\left(P_{0} 1\right)(r, 0) \leq C$ for some universal constant, so that $\left|\left(\mathcal{P}_{0} a\right)(r, \theta)\right| \leq C \int_{\mathbb{T}}|a(\phi)| d \phi \leq C$. In either case

$$
\sup _{|\theta|>2 s}\left|\left(\mathcal{P}_{0} a\right)(r, \theta)\right| d \theta \leq C .
$$

Proof of Proposition 2.1. Let $f \in H^{1}(\mathbb{T})$. Then $f$ has an atomic decomposition $f=\sum_{k \geq 0} \lambda_{k} a_{k}$, converging in $L^{1}(\mathbb{T})$, where $\left(a_{k}\right)_{k \geq 0}$ are atoms and the weights satisfy $\sum_{k \geq 0}\left|\lambda_{k}\right|<+\infty$.

Suppose that we have proved that

$$
\left(\mathcal{P}_{0}^{*} f\right)(\theta) \leq \sum_{k \geq 0}\left|\lambda_{k}\right| \cdot\left(\mathcal{P}_{0}^{*} a_{k}\right)(\theta)
$$

almost everywhere. Then by monotone convergence and Lemma 4.1 we get

$$
\left\|\mathcal{P}_{0}^{*} f\right\|_{L^{1}(\mathbb{T})} \leq \sum_{k \geq 0}\left|\lambda_{k}\right| \cdot\left\|\mathcal{P}_{0}^{*} a_{k}\right\|_{L^{1}(\mathbb{T})} \leq C \sum_{k \geq 0}\left|\lambda_{k}\right|,
$$

where $C$ does not depend on $f$, and taking the infimum over all atomic representations of $f$ yields the proposition. Hence it only remains to prove inequality (4.1). The proof we give carries through for every weak type $(1,1)$ operator $T$ that pointwise satisfies $|T(f+g)| \leq|T f|+|T g|$ and $|T(\lambda f)| \leq$ $|\lambda| \cdot|T f|$ for all functions $f, g$ and scalars $\lambda \in \mathbb{R}$. That the radial maximal function $\mathcal{P}_{0}^{*}$ is of weak type $(1,1)$ was proved in [23] in fact this was shown even for the weakly tangential maximal function.

We have, for $N \in \mathbb{N}$,

$$
\begin{aligned}
\left(\mathcal{P}_{0}^{*} f\right)(\theta) & =\left(\mathcal{P}_{0}^{*} \sum_{k \geq 0} \lambda_{k} a_{k}\right)(\theta)=\sup _{r \in[0,1[}\left|\left(\mathcal{P}_{0} \sum_{k \geq 0} \lambda_{k} a_{k}\right)(r, \theta)\right| \\
& \leq \sup _{r \in[0,1[}\left(\sum_{k=0}^{N}\left|\lambda_{k}\right| \cdot\left|\left(\mathcal{P}_{0} a_{k}\right)(r, \theta)\right|+\left|\left(\mathcal{P}_{0} \sum_{k>N} \lambda_{k} a_{k}\right)(r, \theta)\right|\right) \\
& \leq \sum_{k=0}^{N}\left|\lambda_{k}\right| \cdot\left(\mathcal{P}_{0}^{*} a_{k}\right)(\theta)+\left(\mathcal{P}_{0}^{*} \sum_{k>N} \lambda_{k} a_{k}\right)(\theta) .
\end{aligned}
$$


Given $\epsilon>0$, we deduce by the weak type $(1,1)$ estimate that

$$
\left|\left\{\theta \in \mathbb{T} ;\left(\mathcal{P}_{0}^{*} \sum_{k>N} \lambda_{k} a_{k}\right)(\theta) \geq \epsilon\right\}\right| \leq \frac{C}{\epsilon}\left\|\sum_{k>N} \lambda_{k} a_{k}\right\|_{L^{1}(\mathbb{T})},
$$

which converges to zero as $N \rightarrow+\infty$, that is,

$$
\mathcal{P}_{0}^{*} \sum_{k>N} \lambda_{k} a_{k} \rightarrow 0 \quad \text { in measure }
$$

as $N \rightarrow+\infty$. Consequently there exists a subsequence $\left(N_{j}\right)_{j \geq 1}$ such that $\mathcal{P}_{0}^{*} \sum_{k>N_{j}} \lambda_{k} a_{k} \rightarrow 0$ almost everywhere as $j \rightarrow+\infty$. Hence inequality 4.1 holds, and the proposition follows.

4.2. Decreasing functions and positive functions. Clearly, Proposition 2.2 implies that $H_{\sqrt{ }}^{1}(\mathbb{T})$ is strictly larger than its classical counterpart $H^{1}(\mathbb{T})$. For example, fix $\left.\left.p \in\right] 1,2\right]$ and let

$$
f(\phi)=\frac{1}{\phi \log (1 / \phi)^{p}}
$$

for sufficiently small $\phi>0$, and zero otherwise. Then $f$ is a positive and decreasing function, and we see that $f \in L \log \log L(\mathbb{T}) \backslash L \log L(\mathbb{T})$. Hence by the proposition and the classical result, $f \in H_{\sqrt{ }}^{1}(\mathbb{T}) \backslash H^{1}(\mathbb{T})$.

Proof of Proposition 2.2. Take $f \in L^{1}(\mathbb{T})$ as in the statement of the proposition.

Without loss of generality, we may assume that $\zeta=0$. We may also assume that $f$ is positive, as we see by decomposing the function into its positive and negative parts, $f=f^{+}-f^{-}$. Now $f^{+}$is positive and decreasing from 0 , and $f^{-}$is positive and decreasing from $\pi$, and obviously $f \in L \log \log L(\mathbb{T})$ if and only if $f^{+}, f^{-} \in L \log \log L(\mathbb{T})$. Furthermore, $f \in H_{\sqrt{V}}^{1}(\mathbb{T})$ if and only if $f^{+}, f^{-} \in H_{\sqrt{V}}^{1}(\mathbb{T})$, which is easy to see since any singularities of $f^{+}$and $f^{-}$are separated, in fact even antipodal. Finally, we may assume that the support of $f$ is contained in $[0, \pi]$; the general case follows easily from this special case.

Assume first that $f \in L \log \log L(\mathbb{T})$. In the simple estimate

$$
\begin{aligned}
\left(\mathcal{P}_{0}^{*} f\right)(\theta) & \leq \sup _{r \in[0,2 / 3]}\left(\mathcal{P}_{0} f\right)(r, \theta)+\sup _{r \in] 2 / 3,1[}\left(\mathcal{P}_{0} f\right)(r, \theta) \\
& \leq C\|f\|_{L^{1}(\mathbb{T})}+C \sup _{r \in] 2 / 3,1[} \frac{1}{\log \left(\frac{1}{1-r}\right)} \int_{\mathbb{T}} \frac{f(\phi) d \phi}{(1-r)+|\theta-\phi|},
\end{aligned}
$$

the first term is obviously in $L^{1}(\mathbb{T})$. 
Let $t \in] 0,1 / 3[$. For almost all $\theta$ we have

$$
\begin{aligned}
\frac{1}{\log (1 / t)} \int_{\mathbb{T}} \frac{f(\phi) d \phi}{t+|\theta-\phi|} & =\frac{1}{\log (1 / t)} \int_{0}^{|\theta| / 2} \frac{f(\phi) d \phi}{t+|\theta-\phi|}+\frac{1}{\log (1 / t)} \int_{|\theta| / 2}^{\pi} \frac{f(\phi) d \phi}{t+|\theta-\phi|} \\
& \leq \frac{1}{\log (1 / t)} \cdot \frac{1}{t+|\theta| / 2} \int_{0}^{|\theta| / 2} f(\phi) d \phi+C f(|\theta| / 2) \\
& \leq \frac{2}{|\theta| \log (2 /|\theta|)} \int_{0}^{|\theta| / 2} f(\phi) d \phi+C f(|\theta| / 2),
\end{aligned}
$$

where the last inequality follows by considering separately the cases $|\theta| / 2<$ $t<1 / 3$, in which case $t \mapsto t \log (1 / t)$ is increasing, and $t \leq|\theta| / 2$. Hence, for almost all $\theta$,

$$
\left(\mathcal{P}_{0}^{*} f\right)(\theta) \leq C\|f\|_{L^{1}(\mathbb{T})}+C f(|\theta| / 2)+\frac{2}{|\theta| \log (2 /|\theta|)} \int_{0}^{|\theta| / 2} f(\phi) d \phi,
$$

where, of course, the first two terms are integrable. As for the third term, we see that it is obviously integrable over large $\theta$, say for $|\theta|>2 / e$. For the remaining range, $|\theta| \leq 2 / e$, we have

$$
\int_{|\theta| \leq 2 / e} \frac{1}{|\theta| \log (2 /|\theta|)} \int_{\phi=0}^{|\theta| / 2} f(\phi) d \phi d \theta=2 \int_{0}^{1 / e} f(\phi) \log \log (1 / \phi) d \phi .
$$

Now let $g(\phi)=f(\phi)+1 / \sqrt{\phi}, 0<\phi \leq 1 / e$, which, being a finite sum of $L \log \log L(\mathbb{T})$ functions, satisfies the condition $\int_{0}^{1 / e} \Phi(g(\phi)) d \phi<+\infty$ for $\Phi(x)=x \log (1+\log (1+x)), x \geq 0$. Since $1 / \sqrt{\phi} \leq g(\phi)$, taking logarithms gives

$$
\log \log (1 / \phi) \leq \log \log g(\phi)+\log 2 \leq \log (1+\log (1+g(\phi)))+\log 2,
$$

so that

$$
\int_{0}^{1 / e} f(\phi) \log \log (1 / \phi) d \phi \leq \int_{0}^{1 / e} \Phi(g(\phi)) d \phi+C\|f\|_{L^{1}(\mathbb{T})}<+\infty .
$$

Hence $f \in H_{\sqrt{ }}^{1}(\mathbb{T})$ and we have shown that $L \log \log L(\mathbb{T}) \subseteq H_{\sqrt{ }}^{1}(\mathbb{T})$ when restricted to positive and decreasing functions.

Conversely, suppose that $f \in H_{\sqrt{V}}^{1}(\mathbb{T})$. Without loss of generality we may assume that $\|f\|_{L^{1}(\mathbb{T})} \leq 1$. Let $\left.t \in\right] 0,1 / 2[$ and $|\theta|<1$. Then

$$
\left(\mathcal{P}_{0}^{*} f\right)(\theta) \geq \frac{c}{\log (1 / t)} \int_{0}^{|\theta| / 2} \frac{f(\phi) d \phi}{t+|\theta-\phi|} \geq \frac{c}{\log (1 / t)} \int_{0}^{|\theta| / 2} \frac{f(\phi) d \phi}{t+3|\theta| / 2},
$$


and choosing $t=|\theta| / 2$ we get

$$
\left(\mathcal{P}_{0}^{*} f\right)(\theta) \geq \frac{c}{\log (2 /|\theta|)} \int_{0}^{|\theta| / 2} \frac{f(\phi) d \phi}{2|\theta|} .
$$

Since $\mathcal{P}_{0}^{*} f \in L^{1}(\mathbb{T})$ we may integrate over $\theta \in \mathbb{T}$ with $|\theta|<2 / e$ and get

$$
+\infty>\int_{|\theta|<2 / e} \frac{1}{|\theta| \log (2 /|\theta|)} \int_{\phi=0}^{|\theta| / 2} f(\phi) d \phi d \theta=2 \int_{0}^{1 / e} f(\phi) \log \log (1 / \phi) d \phi .
$$

Now $f(\phi) \leq 1 / \phi$ for almost all $\phi \in] 0, \pi]$, since

$$
1 \geq\|f\|_{L^{1}(\mathbb{T})} \geq \int_{0}^{\phi} f(\theta) d \theta \geq f(\phi) \phi
$$

for almost all $\phi$ in this interval. Thus

$$
\log (1+\log (1+f(\phi))) \leq \log (1+\log (1+1 / \phi)) \leq C \log \log (1 / \phi)
$$

where the last inequality holds for $\phi \in[0,1 / 3]$, say, so that

$$
\int_{0}^{1 / 3} f(\phi) \log (1+\log (1+f(\phi))) d \phi \leq C \int_{0}^{1 / 3} f(\phi) \log \log (1 / \phi) d \phi<+\infty .
$$

Since $f \in L^{1}(\mathbb{T})$ is decreasing, we may extend the integration to all of $\mathbb{T}$ and get $f \in L \log \log L(\mathbb{T})$, which completes the proof of Proposition 2.2.

We now turn to the proof of Theorem 2.3, showing that both implications in Proposition 2.2 may fail for positive functions which are not decreasing, even if we consider a smaller or a larger Orlicz space. In the proof we will construct the functions required for parts (i) and (ii) by forming linear combinations of indicator functions $1_{E}$. The weight $\alpha$ for $1_{E}$ is chosen so that $\Phi(\alpha) \cdot|E|$ is small compared to $\left\|\mathcal{P}_{0}^{*}\left(\alpha 1_{E}\right)\right\|_{L^{1}(\mathbb{T})}$ in part (i), and vice versa in part (ii). The indicator functions we will use are given by the following two lemmas.

LEMMA 4.2. Let $R \geq 0$ be a real number, $\nu \geq 1$ an integer and $J \subseteq \mathbb{T}$ an interval. If $2^{\nu} \geq R^{2}$ and $|J| \geq 2^{-R}$, then there exists a set $E \subseteq J$ with measure $|E|=2^{-\nu-R-1}$ and whose indicator function $1_{E}$ satisfies

$$
\int_{J}\left(\mathcal{P}_{0}^{*} 1_{E}\right)(\theta) d \theta \geq c \nu 2^{-\nu-R}
$$

where $c$ does not depend on $\nu, R$, or $J$.

LEMMA 4.3. Let $R \geq 0, \nu \geq 1$ be real numbers and $J \subseteq \mathbb{T}$ an interval. If $2^{2^{\nu}-R}$ is a strictly positive integer and $|J| \geq 2^{-R}$, then there exists a set $E \subseteq J$ with measure $|E|=2^{-2^{\nu}-R}$ and whose indicator function $1_{E}$ satisfies $\left\|\mathcal{P}_{0}^{*} 1_{E}\right\|_{L^{1}(\mathbb{T})} \leq C 2^{-2^{\nu}}$, where $C$ does not depend on $\nu, R$, or $J$. 
Proof of Lemma 4.2. Without loss of generality we may assume that $\nu \geq 4$ and that $J=\left\{\theta \in \mathbb{T} ; 0 \leq \theta \leq 2^{-R}\right\}$. For $\left.f \in L^{1}(\mathbb{T}), t \in\right] 0,1 / 2[$ and $\theta \in \mathbb{T}$ we let

$$
\begin{aligned}
(\mathcal{K} f)(t, \theta) & =\frac{1}{\log _{2}(1 / t)} \int_{|\theta-\phi|<1 / 2} \frac{f(\phi) d \phi}{t+|\theta-\phi|}, \\
\left(\mathcal{K}^{*} f\right)(\theta) & =\sup _{t \in] 0,1 / 2[}|(\mathcal{K} f)(t, \theta)| .
\end{aligned}
$$

Note that $\mathcal{K}^{*} f$ is measurable, and that $\left(\mathcal{P}_{0}^{*} f\right)(\theta) \geq c\left(\mathcal{K}^{*} f\right)(\theta)$ if $f \in L^{1}(\mathbb{T})$ with $f \geq 0$.

We now recursively define dyadic sets $K_{\mu}$ and $I_{\mu}, \mu \in \mathbb{N}$, such that at step $\mu$, the set $K_{\mu}$ is divided into intervals of length $2^{-R-2^{\mu+1}}$, with the union of every second interval forming $K_{\mu+1}$, and the union of the rest forming $I_{\mu+1}$. More precisely, we let $K_{0}=\left[0,2^{-R-1}\right], I_{0}=\left[2^{-R-1}, 2^{-R}\right]$ and for $\mu \in \mathbb{N}^{+}$,

$$
K_{\mu}=\bigcup_{0 \leq k<2^{2^{\mu}-1}}\left[\frac{2 k}{2^{2^{\mu}+R}}, \frac{2 k+1}{2^{2^{\mu}+R}}\right] \cap K_{\mu-1}, \quad I_{\mu}=\overline{K_{\mu-1} \backslash K_{\mu}} .
$$

Then $K_{\mu}=K_{\mu, 1} \cup \cdots \cup K_{\mu, N_{\mu}}$ and $I_{\mu}=I_{\mu, 1} \cup \cdots \cup I_{\mu, N_{\mu}}$, where $K_{\mu, k}=$ $\left[2 k / 2^{2^{\mu}+R},(2 k+1) / 2^{2^{\mu}+R}\right]$ and $I_{\mu, k}=\left[(2 k-1) / 2^{2^{\mu}+R}, 2 k / 2^{2^{\mu}+R}\right]$ (Fig. 1 ). The number of connected components in $K_{\mu}$ or $I_{\mu}$ is $N_{\mu}=2^{2^{\mu}-\mu-1}$, but we will not need this fact.

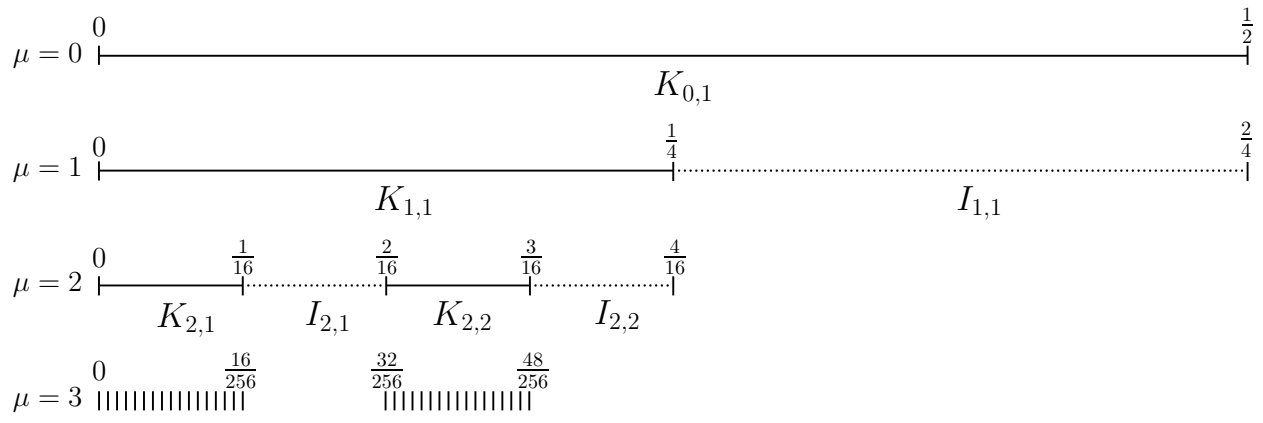

Fig. 1. Level $\mu$ consists of $2^{2^{\mu}-\mu-1}$ intervals of each type, $K$ and $I$, each having length $2^{-R-2^{\mu}}$, where in the figure $R=0$. The set $E$ is chosen as $K_{\nu}$, the union of the $K$ intervals at level $\nu$, and the integral of $\mathcal{P}_{0}^{*} 1_{E}$ will be estimated from below by integrating over $I$-intervals up to level $\nu$.

Let $E=K_{\nu}$, which is a measurable subset of $J$ with $|E|=2^{-\nu-R-1}$, and it remains to prove the desired lower bound on $\int_{J}\left(\mathcal{P}_{0}^{*} 1_{E}\right)(\theta) d \theta$. For this we fix $\mu \in \mathbb{N}$ such that $\nu / 2 \leq \mu<\nu$, and assume that $\theta \in I_{\mu}$. Letting $k$ be such 
that $K_{\mu-1, k} \supseteq I_{\mu}$ we have

$$
\begin{aligned}
\left(\mathcal{K}^{*} 1_{E}\right)(\theta) & \geq\left(\mathcal{K} 1_{E}\right)\left(2^{-R-2^{\mu}}, \theta\right) \geq\left(R+2^{\mu}\right)^{-1} \int_{K_{\mu} \cap K_{\mu-1, k}} \frac{1_{E}(\phi) d \phi}{2^{-R-2^{\mu}+|\theta-\phi|}} \\
& =\left(R+2^{\mu}\right)^{-1} \sum_{i} \int_{K_{\mu, i}} \frac{1_{E}(\phi) d \phi}{2^{-R-2^{\mu}}+|\theta-\phi|},
\end{aligned}
$$

where the sum is over those $i$ for which $K_{\mu, i} \subseteq K_{\mu-1, k}$. In the integral over $K_{\mu, i}$ we may - at the cost of a constant - replace $1_{E}$ by its average value $2^{\mu-\nu}$ over $K_{\mu, i}$, using the fact that the kernel $1 /\left(2^{-R-2^{\mu}}+|\theta-\phi|\right)$ varies no more than by a factor of two when $\phi$ varies in $K_{\mu, i}$ and $\theta$ is fixed. Thus we find that

$$
\begin{aligned}
\left(\mathcal{K}^{*} 1_{E}\right)(\theta) & \geq c\left(R+2^{\mu}\right)^{-1} \cdot 2^{\mu-\nu} \int_{K_{\mu} \cap K_{\mu-1, k}} \frac{d \phi}{2^{-R-2^{\mu}}+|\theta-\phi|} \\
& \geq c\left(R+2^{\mu}\right)^{-1} \cdot 2^{\mu-\nu} \int_{K_{\mu-1, k}} \frac{d \phi}{2^{-R-2^{\mu}}+|\theta-\phi|},
\end{aligned}
$$

where, again at the cost of a constant, the integration has been extended to all of $K_{\mu-1, k}$. Since $\theta \in K_{\mu-1, k}$ the quantity $|\theta-\phi|$ will vary at least from 0 to $\left|K_{\mu-1, k}\right| / 2=2^{-R-2^{\mu-1}-1}$ when we integrate over $\phi \in K_{\mu-1, k}$, so that

$$
\begin{aligned}
\left(\mathcal{K}^{*} 1_{E}\right)(\theta) & \geq c\left(R+2^{\mu}\right)^{-1} \cdot 2^{\mu-\nu} \int_{0}^{2^{-R-2^{\mu-1}-1}} \frac{d \psi}{2^{-R-2^{\mu}+\psi}} \\
& \geq c\left(R+2^{\mu}\right)^{-1} \cdot 2^{\mu-\nu} \cdot 2^{\mu} \geq c 2^{\mu-\nu},
\end{aligned}
$$

where the last inequality follows since $2^{\nu} \geq R^{2}$ and we have assumed that $\mu \geq \nu / 2$. Integration over $I_{\mu}$, which has measure $\left|I_{\mu}\right|=2^{-R-\mu-1}$, gives

$$
\int_{I_{\mu}}\left(\mathcal{K}^{*} 1_{E}\right)(\theta) d \theta \geq c 2^{\mu-\nu} \cdot 2^{-R-\mu-1} .
$$

Finally, using the fact that $\left(I_{\mu}\right)_{\mu \geq 0}$ have pairwise disjoint interiors, we sum over $\mu$ such that $\nu / 2 \leq \mu<\nu$ to get

$$
\int_{J}\left(\mathcal{K}^{*} 1_{E}\right)(\theta) d \theta \geq c 2^{-\nu-R} \cdot \nu
$$

which finishes the proof of Lemma 4.2 .

Proof of Lemma 4.3. Without loss of generality we may assume that $J=\left\{\theta \in \mathbb{T} ; 0 \leq \theta \leq 2^{-R}\right\}$. Let the operators $\mathcal{K}$ and $\mathcal{K}^{*}$ be as in the proof of Lemma 4.2. Henceforth we consider only positive $f \in L^{1}(\mathbb{T}), f \geq 0$. Note that $\mathcal{K}^{*} f$ is measurable and that

$$
\left(\mathcal{P}_{0}^{*} f\right)(\theta) \leq C\|f\|_{L^{1}(\mathbb{T})}+C\left(\mathcal{K}^{*} f\right)(\theta) .
$$


We have

$$
\left(\mathcal{K}^{*} f\right)(\theta) \leq C \sup _{\mu \in \mathbb{N}^{+}}(\mathcal{K} f)\left(2^{-2^{\mu}}, \theta\right),
$$

since clearly $\left(\mathcal{K}^{*} f\right)(\theta) \leq C \sup _{N \in \mathbb{N}^{+}}(\mathcal{K} f)\left(2^{-N}, \theta\right)$, and if $2^{\mu-1} \leq N<2^{\mu}$, then

$$
\begin{aligned}
(\mathcal{K} f)\left(2^{-N}, \theta\right) & =\frac{1}{N} \int_{|\theta-\phi|<1 / 2} \frac{f(\phi) d \phi}{2^{-N}+|\theta-\phi|} \\
& \leq \frac{1}{2^{\mu-1}} \int_{|\theta-\phi|<1 / 2} \frac{f(\phi) d \phi}{2^{-2^{\mu}}+|\theta-\phi|}=2 \cdot(\mathcal{K} f)\left(2^{-2^{\mu}}, \theta\right) .
\end{aligned}
$$

Furthermore, $\left(\mathcal{K}^{*} f\right)(\theta) \leq C \sup _{j \geq 1}\left(\mathcal{L}_{j} f\right)(\theta)$, where

$$
\left(\mathcal{L}_{j} f\right)(\theta)=\frac{1}{2^{j}} \int_{|\theta-\phi|<2^{-2^{j-1}}} \frac{f(\phi) d \phi}{2^{-2^{j}}+|\theta-\phi|}, \quad j \in \mathbb{N}^{+},
$$

since

$$
\begin{aligned}
2^{\mu} \cdot(\mathcal{K} f) & \left(2^{-2^{\mu}}, \theta\right)=\int_{|\theta-\phi|<1 / 2} \frac{f(\phi) d \phi}{2^{-2^{\mu}}+|\theta-\phi|} \\
& =\sum_{j=1}^{\mu-1} \int_{2^{-2^{j}} \leq|\theta-\phi|<2^{-2^{j-1}}} \frac{f(\phi) d \phi}{2^{-2^{\mu}+|\theta-\phi|}}+\int_{|\theta-\phi|<2^{-2^{\mu-1}}} \frac{f(\phi) d \phi}{2^{-2^{\mu}}+|\theta-\phi|} \\
& \leq \sum_{j=1}^{\mu-1} \int_{2^{-2^{j}} \leq|\theta-\phi|<2^{-2^{j-1}}} \frac{f(\phi) d \phi}{|\theta-\phi|}+\int_{|\theta-\phi|<2^{-2^{\mu-1}}} \frac{f(\phi) d \phi}{2^{-2^{\mu}}+|\theta-\phi|} \\
& \leq 2 \sum_{j=1}^{\mu} 2^{j} \cdot\left(\mathcal{L}_{j} f\right)(\theta) .
\end{aligned}
$$

Let $M=2^{2^{\nu}-R}$, where $\nu$ and $R$ are as in the statement of the lemma, and

$$
E=\bigcup_{k=0}^{M-1}\left[k 2^{-2^{\nu}}, k 2^{-2^{\nu}}+2^{-2^{\nu+1}}\right]
$$

which is a measurable subset of $J$ having measure $|E|=2^{-2^{\nu}-R}$.

We now estimate $\mathcal{L}_{j} 1_{E}, j \in \mathbb{N}^{+}$, and consider first the case when $j \leq\lfloor\nu\rfloor$. In this case the kernel of $\mathcal{L}_{j}$ satisfies $\left(2^{-2^{j}}+|\psi+\delta|\right)^{-1} \sim\left(2^{-2^{j}}+|\psi|\right)^{-1}$ for $\psi \in \mathbb{T}$ and $|\delta| \leq 2^{-2^{\nu}}$, so that

$$
\left(\mathcal{L}_{j} 1_{E}\right)(\theta) \leq \frac{1}{2^{j}} \int_{\mathbb{T}} \frac{1_{E}(\phi) d \phi}{2^{-2^{j}}+|\theta-\phi|}=2^{-j} \sum_{k=0}^{M-1} \int_{k 2^{-2^{\nu}}}^{k 2^{-2^{\nu}}+2^{-2^{\nu+1}}} \frac{d \phi}{2^{-2^{j}}+|\theta-\phi|}
$$




$$
\begin{aligned}
& \leq C 2^{-j} \sum_{k=0}^{M-1} 2^{-2^{\nu+1}+2^{\nu}} \int_{k 2^{-2^{\nu}}}^{(k+1) 2^{-2^{\nu}}} \frac{d \phi}{2^{-2^{j}}+|\theta-\phi|} \\
& =C 2^{-2^{\nu}-j} \int_{0}^{2^{-R}} \frac{d \phi}{2^{-2^{j}}+|\theta-\phi|} \leq C 2^{-2^{\nu}-j} \int_{0}^{2^{-R}} \frac{d \psi}{2^{-2^{j}}+\psi} \\
& =C 2^{-2^{\nu}-j} \log \left(1+2^{2^{j}-R}\right) \leq C 2^{-2^{\nu}} .
\end{aligned}
$$

Consider now the case $j \geq\lfloor\nu\rfloor+3$. Obviously $\left(\mathcal{L}_{j} 1_{E}\right)(\theta) \leq C$, where $C$ does not depend on $j$, and since $\left(\mathcal{L}_{j} 1_{E}\right)(\theta)=0$ if $\operatorname{dist}(\theta, E) \geq 2^{-2^{\nu+1}}$ we see that $\left(\mathcal{L}_{j} 1_{E}\right)(\theta) \leq C 1_{\widetilde{E}}(\theta)$ for all $\theta \in \mathbb{T}$, where again $C$ does not depend on $j$, and

$$
\widetilde{E}=\bigcup_{k=0}^{M-1}\left[k 2^{-2^{\nu}}-2^{-2^{\nu+1}}, k 2^{-2^{\nu}}+2 \cdot 2^{-2^{\nu+1}}\right] .
$$

Thus $\left(\mathcal{L}_{j} 1_{E}\right)(\theta) \leq C\left(2^{-2^{\nu}}+1_{\widetilde{E}}(\theta)\right)$ for all $j \in \mathbb{N}^{+}$except $j=\lfloor\nu\rfloor+1$ and $j=\lfloor\nu\rfloor+2$. By inequality 4.2 we then get

$$
\begin{aligned}
\left\|\mathcal{P}_{0}^{*} 1_{E}\right\|_{L^{1}(\mathbb{T})} & \leq C|E|+C\left(2^{-2^{\nu}}+|\widetilde{E}|\right)+\left\|\mathcal{L}_{\lfloor\nu\rfloor+1} 1_{E}\right\|_{L^{1}(\mathbb{T})}+\left\|\mathcal{L}_{\lfloor\nu\rfloor+2} 1_{E}\right\|_{L^{1}(\mathbb{T})} \\
& \leq C 2^{-2^{\nu}},
\end{aligned}
$$

since $\left\|\mathcal{L}_{j} 1_{E}\right\|_{L^{1}(\mathbb{T})} \leq C\left\|1_{E}\right\|_{L^{1}(\mathbb{T})}$ by Young's inequality for convolutions, where $C$ does not depend on $j=\lfloor\nu\rfloor+1,\lfloor\nu\rfloor+2$. The proof of Lemma 4.3 is complete.

REMARK. The referee made the interesting observation that the constructions in the proofs of Lemmas 4.2 and 4.3 are, quite unexpectedly, similar to the constructions used to prove the Talagrand example for ball packings (see [11]).

Proof of Theorem 2.3. Let $\phi(x)=\Phi(x) / x$ for $x>0$.

(i) There exists a continuous function $\psi$ such that $\psi(t) 2^{-2 \psi(t)}=t$ for all sufficiently small $t>0$, and $\psi(t) \rightarrow+\infty$ as $t \rightarrow 0^{+}$. In fact, $\psi(t)=$ $-a W_{-1}(-b t)$, where $a, b>0$ are constants and $W_{-1}$ is a branch of the Lambert $W$ function (see [7]).

Since $\liminf \operatorname{in}_{x \rightarrow+\infty} \phi(x) / \log x=0$ by assumption, we may for each $k \in$ $\mathbb{N}^{+}$pick $\alpha_{k}>1$ such that $\phi\left(\alpha_{k}\right) / \log \alpha_{k} \leq 1 / k$. We also assume that $\alpha_{k}$ is chosen so large that $\psi\left(\frac{1}{\alpha_{k} k}\right) \geq k$. Let $\nu_{k}=\left\lfloor\psi\left(\frac{1}{\alpha_{k} k}\right)\right\rfloor$ and $R_{k}=\psi\left(\frac{1}{\alpha_{k} k}\right)$. Since $\sum_{k \geq 1} 2^{-R_{k}}<2 \pi$, we may pick pairwise disjoint intervals $\left(J_{k}\right)_{k \geq 1}$ with $\left|J_{k}\right|=2^{-\bar{R}_{k}}$. Using Lemma 4.2 with $J=J_{k}, \nu=\nu_{k}$ and $R=R_{k}$-which for $k \geq 7$ clearly satisfy the conditions of the lemma-we get subsets $E_{k}$ of $J_{k}$ satisfying $\left|E_{k}\right|=2^{-\nu_{k}-R_{k}-1}$ and $\int_{J_{k}}\left(\mathcal{P}_{0}^{*} 1_{E_{k}}\right)(\theta) d \theta \geq c \nu_{k} 2^{-\nu_{k}-R_{k}}$. 
Now let $f=\sum_{k \geq 7} \alpha_{k} 1_{E_{k}}$, which is measurable, and note that, since $\alpha_{k}=2^{2 R_{k}} /\left(R_{k} k\right)$ by the definition of $R_{k}$ and $\psi$,

$$
\begin{aligned}
\int_{\mathbb{T}} \Phi(f(\theta)) d \theta & =\sum_{k \geq 7} \alpha_{k} \phi\left(\alpha_{k}\right) \cdot 2^{-\nu_{k}-R_{k}-1} \\
& \leq \sum_{k \geq 7} \alpha_{k} \cdot \frac{\log \alpha_{k}}{k} \cdot 2^{-\nu_{k}-R_{k}-1} \\
& =\sum_{k \geq 7} \frac{2^{2 R_{k}}}{R_{k} k} \cdot \frac{1}{k} \cdot \log \left(\frac{2^{2 R_{k}}}{R_{k} k}\right) \cdot 2^{-\nu_{k}-R_{k}-1} \leq \sum_{k \geq 7} \frac{C}{k^{2}}<+\infty,
\end{aligned}
$$

and hence $f \in L^{\Phi}(\mathbb{T})$. On the other hand,

$$
\begin{aligned}
\int_{\mathbb{T}}\left(\mathcal{P}_{0}^{*} f\right)(\theta) d \theta & \geq \sum_{k \geq 7} \int_{J_{k}} \alpha_{k}\left(\mathcal{P}_{0}^{*} 1_{E_{k}}\right)(\theta) d \theta \geq c \sum_{k \geq 7} \alpha_{k} \nu_{k} 2^{-\nu_{k}-R_{k}} \\
& \geq c \sum_{k \geq 7} \frac{1}{k}=+\infty,
\end{aligned}
$$

so that $f \notin H_{\sqrt{ }}^{1}(\mathbb{T})$.

(ii) Since $\lim \sup _{x \rightarrow+\infty} \phi(x)=+\infty$ by assumption, we may for each $k \in \mathbb{N}^{+}$pick $\alpha_{k}>4$ such that $\phi\left(\alpha_{k}\right) \geq k^{3}$. Let $\nu_{k}=\log _{2} \log _{2}\left(k^{2} \alpha_{k}\right)$ and $R_{k}=2 \log _{2}(k)-\epsilon_{k}$, where $\epsilon_{k} \in\left[0,1\left[\right.\right.$ is chosen so that $2^{\nu_{k}}-R_{k} \in \mathbb{N}$. Since $\sum_{k>1} 2^{-R_{k}}<2 \pi$ we may pick pairwise disjoint intervals $\left(J_{k}\right)_{k \geq 1}$ with $\left|J_{k}\right|=$ $2^{-R_{k}}$. Using Lemma 4.3 with $J=J_{k}, \nu=\nu_{k}$ and $R=R_{k}$-which clearly satisfy the conditions of the lemma-we get subsets $E_{k}$ of $J_{k}$ satisfying $\left|E_{k}\right|=2^{-2^{\nu} k-R_{k}}$ and $\left\|\mathcal{P}_{0}^{*} 1_{E_{k}}\right\|_{L^{1}(\mathbb{T})} \leq C 2^{-2^{\nu} k}$.

Let $g=\sum_{k \geq 1} \alpha_{k} 1_{E_{k}}$, which is clearly integrable, and note that $g \in$ $H_{\sqrt{ }}^{1}(\mathbb{T})$, since

$$
\left\|\mathcal{P}_{0}^{*} g\right\|_{L^{1}(\mathbb{T})} \leq \sum_{k \geq 1} \alpha_{k}\left\|\mathcal{P}_{0}^{*} 1_{E_{k}}\right\|_{L^{1}(\mathbb{T})} \leq C \sum_{k \geq 1} \alpha_{k} 2^{-2^{\nu_{k}}}=C \sum_{k \geq 1} \frac{1}{k^{2}}<+\infty,
$$

where the first inequality follows by monotone convergence. However,

$$
\int_{\mathbb{T}} \Phi(g(\theta)) d \theta=\sum_{k \geq 1} \alpha_{k} \phi\left(\alpha_{k}\right) \cdot 2^{-2^{\nu} k-R_{k}} \geq \sum_{k \geq 1} \alpha_{k} \cdot k^{3} \cdot \frac{1}{k^{2} \alpha_{k}} \cdot \frac{2^{\epsilon_{k}}}{k^{2}} \geq \sum_{k \geq 1} \frac{1}{k}=+\infty,
$$

so that $g \notin L^{\Phi}(\mathbb{T})$, since $L^{\Phi}(\mathbb{T})$ is of class $\Delta_{2}$.

4.3. Distributions and eigenfunctions. We defined $H_{\sqrt{V}}^{1}(\mathbb{T})$ to be the set of $f \in L^{1}(\mathbb{T})$ for which $\mathcal{P}_{0}^{*} f \in L^{1}(\mathbb{T})$. However, just as in the classical case of $H^{1}(\mathbb{T})$, this space does not change if we a priori allow $f$ to be a distribution, in the following sense. Given a distribution $T \in \mathcal{D}^{\prime}(\mathbb{T})$ we 
define, for $r \in[0,1[$ and $\theta \in \mathbb{T}$,

$$
\begin{aligned}
\left(P_{0} T\right)(r, \theta) & =T\left(P_{0}(r, \theta-\cdot)\right), \\
\left(\mathcal{P}_{0} T\right)(r, \theta) & =T\left(\mathcal{P}_{0}(r, \theta-\cdot)\right), \\
\left(\mathcal{P}_{0}^{*} T\right)(\theta) & =\sup _{r \in[0,1[}\left|\left(\mathcal{P}_{0} T\right)(r, \theta)\right|,
\end{aligned}
$$

where $\mathcal{P}_{0}(r, \theta)=P_{0}(r, \theta) /\left(P_{0} 1\right)(r, 0)$ is the normalized kernel.

In the proofs of Propositions 2.4 and 2.5 we will use Alaoglu's theorem in the separable case. Let $C(\mathbb{T})$ be the set of continuous functions on $\mathbb{T}$. The set $C(\mathbb{T})^{*}$ of bounded linear functionals on $C(\mathbb{T})$ is identified with the set $M(\mathbb{T})$ of complex Radon measures on $\mathbb{T}$, equipped with the norm $\|\mu\|=|\mu|(\mathbb{T})$, where $|\mu|$ is the total variation of $\mu \in M(\mathbb{T})$. Then for any family $\left(A_{r}\right)_{r \in[0,1[}$ of operators in $C(\mathbb{T})^{*}$ with uniformly bounded operator norm, we may take a sequence $\left(r_{n}\right)_{n \geq 1}$ in $\left[0,1\left[\right.\right.$ and a measure $\mu \in M(\mathbb{T})$ such that $r_{n} \rightarrow 1$ and $A_{r_{n}} \rightarrow \mu$ vaguely as $n \rightarrow+\infty$, that is,

$$
\lim _{n \rightarrow+\infty} A_{r_{n}}(f)=\int_{\mathbb{T}} f(\theta) d \mu(\theta)
$$

for all $f \in C(\mathbb{T})$.

The proof of Proposition 2.4 is essentially the same as in the classical case (see for instance [9, Theorem 6.4.3]). Basically we show that there exists a sequence $\left(r_{n}\right)_{n \geq 1}$ such that $\left(\mathcal{P}_{0} T\right)\left(r_{n}, \cdot\right)$ converges both to $T$ in $\mathcal{D}^{\prime}(\mathbb{T})$ and to some measure, a measure which is then shown to be absolutely continuous with respect to the arc measure on $\mathbb{T}$.

Proof of Proposition 2.4. If $r \in\left[0,1\left[\right.\right.$ is fixed we may interpret $\left(\mathcal{P}_{0} T\right)(r, \cdot)$ as an element in $C(\mathbb{T})^{*}$ by letting, for $f \in C(\mathbb{T})$,

$$
\left(\left(\mathcal{P}_{0} T\right)(r, \cdot)\right)(f)=\int_{\mathbb{T}}\left(\mathcal{P}_{0} T\right)(r, \theta) f(\theta) d \theta,
$$

and we see that $\left|\left(\left(\mathcal{P}_{0} T\right)(r, \cdot)\right)(f)\right| \leq\|f\|_{L^{\infty}(\mathbb{T})}\left\|\mathcal{P}_{0}^{*} T\right\|_{L^{1}(\mathbb{T})}$.

Take $\left(r_{n}\right)_{n \geq 1}$ in $\left[0,1\left[\right.\right.$ and $\mu \in M(\mathbb{T})$ with $r_{n} \rightarrow 1$ and $\left(\mathcal{P}_{0} T\right)\left(r_{n}, \cdot\right) \rightarrow \mu$ vaguely as $n \rightarrow+\infty$. Both the measure $\mu$ and the function $\left(\mathcal{P}_{0} T\right)(r, \cdot)$, for fixed $r \in[0,1$ [, are interpreted as distributions in the usual way. We now proceed to show that $\mu=T$ by first proving that $\left(\mathcal{P}_{0} T\right)(r, \cdot) \rightarrow T$ in $\mathcal{D}^{\prime}(\mathbb{T})$ as $r \rightarrow 1^{-}$. That is, we show that for every $f \in \mathcal{D}(\mathbb{T})$ we have

$$
\lim _{r \rightarrow 1^{-}}\left(\left(\mathcal{P}_{0} T\right)(r, \cdot)\right)(f)=T(f) .
$$

Since $\left(\left(\mathcal{P}_{0} T\right)(r, \cdot)\right)(f)=\left(T * \mathcal{P}_{0}(r, \cdot)\right)(f)=T\left(f * \mathcal{P}_{0}(r, \cdot)\right)$ we only have 
to show that $f * \mathcal{P}_{0}(r, \cdot) \rightarrow f$ in $\mathcal{D}(\mathbb{T})$ as $r \rightarrow 1^{-}$. However, for all $\alpha \in \mathbb{N}$,

$$
\begin{aligned}
\left|\partial^{\alpha}\left(f * \mathcal{P}_{0}(r, \cdot)\right)(\theta)-\partial^{\alpha} f(\theta)\right| & =\left|\left(\left(\partial^{\alpha} f-\partial^{\alpha} f(\theta)\right) * \mathcal{P}_{0}(r, \cdot)\right)(\theta)\right| \\
& \leq \frac{C}{\log \left(\frac{2}{1-r}\right)} \int_{\mathbb{T}} \frac{\left|\partial^{\alpha} f(\phi)-\partial^{\alpha} f(\theta)\right|}{(1-r)+|\theta-\phi|} d \phi \\
& \leq \frac{C}{\log \left(\frac{2}{1-r}\right)} \int_{\mathbb{T}} \frac{\left\|\partial^{\alpha+1} f\right\|_{L^{\infty}(\mathbb{T})} \cdot|\theta-\phi|}{0+|\theta-\phi|} d \phi \\
& =\frac{C\left\|\partial^{\alpha+1} f\right\|_{L^{\infty}(\mathbb{T})}}{\log \left(\frac{2}{1-r}\right)} \rightarrow 0
\end{aligned}
$$

as $r \rightarrow 1^{-}$, and equation 4.3 follows. Hence if $f \in \mathcal{D}(\mathbb{T})$ we see that $\int_{\mathbb{T}} f d \mu=\lim _{n \rightarrow+\infty} \int_{\mathbb{T}}\left(\mathcal{P}_{0} T\right)\left(r_{n}, \theta\right) f(\theta) d \theta=T(f)$, so that $\mu=T$.

It only remains to show that $\mu$ is absolutely continuous with respect to Lebesgue measure. Take $E \subseteq \mathbb{T}$ with $|E|=0$. Fix a closed set $F \subseteq E$ and let $\epsilon>0$. Since $\mathcal{P}_{0}^{*} \mu \in L^{1}(\mathbb{T})$ there exists $\delta>0$ such that $\left|\int_{A} \mathcal{P}_{0}^{*} \mu d \theta\right|<\epsilon$ for all $A \subseteq \mathbb{T}$ with $|A|<\delta$. Let, by outer regularity of Lebesgue measure, $U \supseteq E$ be an open set having $|U|<\delta$. Using Lusin's theorem we get a continuous function $f \in C(\mathbb{T})$ satisfying $|\mu|\left(f \neq 1_{F}\right)<\epsilon,\|f\|_{L^{\infty}(\mathbb{T})} \leq 1$ and $\operatorname{supp} f \subseteq U$.

Now $\mu(F)=\int_{\mathbb{T}} f d \mu+\int_{\mathbb{T}}\left(1_{F}-f\right) d \mu$, where

$$
\left|\int_{\mathbb{T}}\left(1_{F}-f\right) d \mu\right| \leq 2|\mu|\left(f \neq 1_{F}\right)<2 \epsilon,
$$

and

$$
\begin{aligned}
\left|\int_{\mathbb{T}} f d \mu\right| & =\lim _{n \rightarrow+\infty}\left|\int_{\mathbb{T}}\left(\mathcal{P}_{0} \mu\right)\left(r_{n}, \theta\right) f(\theta) d \theta\right| \\
& \leq \limsup _{n \rightarrow+\infty} \int_{\mathbb{T}}\left|\left(\mathcal{P}_{0} \mu\right)\left(r_{n}, \theta\right)\right| \cdot|f(\theta)| d \theta \leq \int_{U}\left(\mathcal{P}_{0}^{*} \mu\right)(\theta) \cdot 1 d \theta<\epsilon,
\end{aligned}
$$

since $|U|<\delta$. Hence $|\mu(F)|<3 \epsilon$, and since $\epsilon>0$ is arbitrary, it follows that $\mu(F)=0$. Noting that $\mu$ and $|\mu|$ have the same null sets, we find that $|\mu|(F)=0$, and by inner regularity $|\mu|(E)=0$, showing that $\mu(E)=0$. Consequently $\mu$ is absolutely continuous with respect to Lebesgue measure, and Proposition 2.4 follows from the Radon-Nikodym theorem.

By [10, Theorem 1.7], any function satisfying condition (i) in Proposition 2.5 can be represented as $P_{0} T$-defined in the obvious way-for an analytic functional $T$. We will not need this general result, but Proposition 2.5 follows easily from its proof. However, for completeness we instead give an essentially self-contained proof of our special case. Although the method of proof is the same as in [10], our proof does not employ the more general theory used there. In passing, we also mention that an eigenfunction 
is represented by a distribution $T \in \mathcal{D}^{\prime}(\mathbb{T})$ if and only if it grows at most exponentially with the hyperbolic distance to the origin [1, 15].

Proof of Proposition 2.5. The converse statement is trivial. On the other hand, if $u \in C^{2}(\mathbb{D})$ satisfies conditions (i) and (ii) of the proposition, then we define a family of bounded linear functionals $A_{r} \in C(\mathbb{T})^{*}, r \in[0,1[$, by

$$
A_{r}(g)=\frac{1}{\left(P_{0} 1\right)(r, 0)} \int_{\mathbb{T}} u(r, \theta) g(\theta) d \theta \quad \text { for } g \in C(\mathbb{T}) .
$$

Now $\left|A_{r}(g)\right| \leq\left\|u^{*}\right\|_{L^{1}(\mathbb{T})}\|g\|_{L^{\infty}(\mathbb{T})}$, so we may take a sequence $\left(r_{k}\right)_{k \geq 1}$ in $\left[0,1\left[\right.\right.$ and a measure $\mu \in M(\mathbb{T})$ such that $r_{k} \rightarrow 1$ and $A_{r_{k}} \rightarrow \mu$ vaguely as $k \rightarrow+\infty$.

Hence to finish the proof we only need to show that $P_{0} \mu=u$, since in that case $d \mu=f d \theta$ for some $f \in H_{\sqrt{ }}^{1}(\mathbb{T})$ by Proposition 2.4 and condition (ii). Expanding $u(r, \cdot)$ in a Fourier series, $u(r, \theta)=\sum_{n} c_{n}(r) e^{i n \theta}$, we first show that there exist constants $a_{n}$ such that $c_{n}(r) e^{i n \theta}=a_{n} f_{n}(r, \theta)$, where

$$
f_{n}(r, \theta)=\frac{1}{2 \pi} \int_{\mathbb{T}} P_{0}(r, \theta-\phi) e^{i n \phi} d \phi .
$$

Note that both $(r, \theta) \mapsto c_{n}(r) e^{i n \theta}=\frac{1}{2 \pi}\left(u(r, \cdot) * e^{i n \cdot}\right)(\theta)$ and $f_{n}$ satisfy

$$
\begin{aligned}
\frac{1}{4}\left(1-r^{2}\right)^{2} \Delta v(r, \theta) & =-\frac{1}{4} v(r, \theta), \\
v(r, \theta) & =e^{i n \theta} v(r, 0) .
\end{aligned}
$$

Also observe that $\left(P_{0}(r, \cdot) /\left(P_{0} 1\right)(r, 0)\right)_{r \in[0,1[}$ is an approximation of the identity, in the sense that for every $g \in C(\mathbb{T})$ we have

$$
\left(\mathcal{P}_{0} g\right)(r, 0)=\frac{1}{\left(P_{0} 1\right)(r, 0)} \int_{\mathbb{T}} P_{0}(r, \phi) g(\phi) d \phi \rightarrow g(0)
$$

as $r \rightarrow 1^{-}$. In fact, for each $\epsilon>0$ there exists $\left.\delta \in\right] 0, \pi[$ such that $|g(\phi)-g(0)|<\epsilon$ for $|\phi| \leq \delta$, thus

$$
\begin{aligned}
\left|\left(\mathcal{P}_{0} g\right)(r, \phi)-g(0)\right| & =\left|\left(\mathcal{P}_{0}(g-g(0))\right)(r, \phi)\right| \\
& \leq \epsilon+\frac{2\|g\|_{L^{\infty}(\mathbb{T})}}{\left(P_{0} 1\right)(r, 0)} \int_{|\phi|>\delta} P_{0}(r, \phi) d \phi \\
& \leq \epsilon+\frac{C_{\delta}\|g\|_{L^{\infty}(\mathbb{T})}}{\log \left(\frac{2}{1-r}\right)},
\end{aligned}
$$

where the last term tends to zero as $r \rightarrow 1^{-}$. Consequently, $f_{n}(R, 0)$ $\neq 0$ for all sufficiently large $R \in] 0,1[$, with $n$ fixed. For such $R$ we define constants $d_{R}$ by $c_{n}(R)=d_{R} f_{n}(R, 0)$. Then $c_{n}(R) e^{i n \theta}=d_{R} f_{n}(R, \theta)$ for all $\theta \in \mathbb{T}$, because of 4.5 . Applying the strong maximum principle 
to $(r, \theta) \mapsto\left(c_{n}(r) e^{i n \theta}-d_{R} f_{n}(r, \theta)\right) / f_{0}(r, \theta)$ (see for instance [17, Theorem 2.10]), we deduce that $c_{n}(r) e^{i n \theta}=d_{R} f_{n}(r, \theta)$ for all $r \in[0, R]$ and $\theta \in \mathbb{T}$. Obviously $d_{R}$ cannot depend on $R$, allowing us to pick $a_{n}=d_{R}$.

Since $P_{0}(r, \theta-\phi)=\sum_{n} f_{n}(r, \theta) e^{-i n \phi}$, it suffices to show $\mu\left(e^{-i n \cdot}\right)=a_{n}$ in order to prove that $P_{0} \mu=u$. However, by the vague convergence,

$$
\begin{aligned}
\mu\left(e^{-i n \cdot}\right) & =\lim _{k \rightarrow+\infty} \frac{1}{\left(P_{0} 1\right)\left(r_{k}, 0\right)} \int_{\mathbb{T}} u\left(r_{k}, \phi\right) e^{-i n \phi} d \phi=\lim _{k \rightarrow+\infty} \frac{2 \pi c_{n}\left(r_{k}\right)}{\left(P_{0} 1\right)\left(r_{k}, 0\right)} \\
& =\lim _{k \rightarrow+\infty} \frac{2 \pi a_{n} f_{n}\left(r_{k}, 0\right)}{\left(P_{0} 1\right)\left(r_{k}, 0\right)}=\lim _{k \rightarrow+\infty} \frac{a_{n}}{\left(P_{0} 1\right)\left(r_{k}, 0\right)} \int_{\mathbb{T}} P_{0}\left(r_{k}, \phi\right) e^{i n \phi} d \phi=a_{n},
\end{aligned}
$$

where the last equality follows from (4.6).

Acknowledgments. The author is grateful to his co-advisor P. Sjögren who suggested the problem and also advised the author during the course of this work. The author would also like to thank the anonymous referee for helpful suggestions.

\section{References}

[1] E. P. van den Ban and H. Schlichtkrull, Asymptotic expansions and boundary values of eigenfunctions on Riemannian symmetric spaces, J. Reine Angew. Math. 380 (1987), 108-165.

[2] M. Brundin, Boundary behaviour of eigenfunctions for the hyperbolic Laplacian, Ph.D. thesis, Chalmers Univ. of Technology and Univ. of Gothenburg, Göteborg, 2002, paper 1 (Approach regions for the square root of the Poisson kernel and weak $L^{p}$ boundary functions).

[3] -, Approach regions for $L^{p}$ potentials with respect to the square root of the Poisson kernel, Math. Scand. 96 (2005), 243-256.

[4] -, Approach regions for the square root of the Poisson kernel and boundary functions in certain Orlicz spaces, Czechoslovak Math. J. 57 (132) (2007), 345-365.

[5] M. Carlsson, Fatou-type theorems for general approximate identities, Math. Scand. 102 (2008), 231-252.

[6] R. R. Coifman and G. Weiss, Extensions of Hardy spaces and their use in analysis, Bull. Amer. Math. Soc. 83 (1977), 569-645.

[7] R. M. Corless, G. H. Gonnet, D. E. G. Hare, D. J. Jeffrey, and D. E. Knuth, On the Lambert $W$ function, Adv. Comput. Math. 5 (1996), 329-359.

[8] J. García-Cuerva and J. L. Rubio de Francia, Weighted Norm Inequalities and Related Topics, North-Holland Math. Stud. 116, North-Holland, Amsterdam, 1985.

[9] L. Grafakos, Classical and Modern Fourier Analysis, Pearson Education, Upper Saddle River, NJ, 2004.

[10] S. Helgason, A duality for symmetric spaces with applications to group representations, Adv. Math. 5 (1970), 1-154.

[11] O. Jørsboe, L. Mejlbro, and F. Topsøe, Some Vitali theorems for Lebesgue measure, Math. Scand. 48 (1981), 259-285. 
[12] F. I. Karpelevich, The geometry of geodesics and the eigenfunctions of the BeltramiLaplace operator on symmetric spaces, Trudy Moskov. Mat. Obshch. 14 (1965), 48-185 (in Russian); English transl.: Trans. Moscow Math. Soc. 14 (1965), 51-199.

[13] J. E. Littlewood, On a theorem of Fatou, J. London Math. Soc. 2 (1927), 172-176.

[14] S. Müller, A surprising higher integrability property of mappings with positive determinant, Bull. Amer. Math. Soc. (N.S.) 21 (1989), 245-248.

[15] T. Ōshima and J. Sekiguchi, Eigenspaces of invariant differential operators on an affine symmetric space, Invent. Math. 57 (1980), 1-81.

[16] M. A. Picardello and P. Sjögren, Boundary behaviour of eigenfunctions of the Laplacian in a bi-tree, J. Reine Angew. Math. 424 (1992), 137-148.

[17] M. H. Protter and H. F. Weinberger, Maximum Principles in Differential Equations, Prentice-Hall, Englewood Cliffs, NJ, 1967.

[18] M. M. Rao and Z. D. Ren, Theory of Orlicz Spaces, Monogr. Textbooks Pure Appl. Math. 146, Dekker, New York, 1991.

[19] J.-O. Rönning, Convergence results for the square root of the Poisson kernel, Math. Scand. 81 (1997), 219-235.

[20] - On convergence for the square root of the Poisson kernel in symmetric spaces of rank 1, Studia Math. 125 (1997), 219-229.

[21] - A convergence result for square roots of the Poisson kernel in the bidisk, Math. Scand. 84 (1999), 81-92.

[22] P. Sjögren, Fatou theorems and maximal functions for eigenfunctions of the LaplaceBeltrami operator in a bidisk, J. Reine Angew. Math. 345 (1983), 93-110.

[23] —, Une remarque sur la convergence des fonctions propres du laplacien à valeur propre critique, in: Théorie du potentiel (Orsay, 1983), Lecture Notes in Math. 1096, Springer, Berlin, 1984, 544-548.

[24] - Approach regions for the square root of the Poisson kernel and bounded functions, Bull. Austral. Math. Soc. 55 (1997), 521-527.

[25] E. M. Stein, Note on the class L $\log L$, Studia Math. 32 (1969), 305-310.

[26] O. Svensson, On generalized Fatou theorems for the square root of the Poisson kernel and in rank one symmetric space, Ann. Scuola Norm. Sup. Pisa Cl. Sci. (4) 23 (1996), 467-482.

Jonatan Vasilis

Department of Mathematical Sciences

Chalmers University of Technology

SE-412 96 Göteborg, Sweden

Department of Mathematical Sciences

University of Gothenburg

E-mail: f00jova@chalmers.se SE-412 96 Göteborg, Sweden

Received October 6, 2009

Revised version April 6, 2010 